\title{
Mechanical properties of glass polymer multilayer composite
}

\author{
A SEAL, $\mathbf{N} R$ bOSE, $S$ K DALUi, A $K$ MUKHOPADHYAY*, $K$ K PHANI and \\ H S MAITI \\ Central Glass and Ceramic Research Institute, Kolkata 700 032, India
}

\begin{abstract}
The preliminary experimental studies on the comparative behaviour of the deformation processes involved in the failure of a commercial, $0.3 \mathrm{~mm}$ thick, $18 \mathrm{~mm}$ diameter soda-lime-silica glass disks $(G)$ and multilayered glass disk-epoxy (GE) as well as glass disk-epoxy- $E$-glass fabric (GEF) composite structures are reported. The failure tests were conducted in a biaxial flexure at room temperature. The epoxy was a commercial resin and the $E$-glass fabric was also commercially obtained as a two-dimensional weave of $E$-glass fibres to an area density of about $242 \mathrm{~g} \mathrm{~m}^{-2}$. The multilayered structures were developed by alternate placement of the glass and reinforcing layers by a hand lay-up technique followed by lamination at an appropriate temperature and pressure. Depending on the number of layers the volume fraction of reinforcement could be varied from about 0.20 for the GE system to about 0.50 for the GEF system. It was observed that the specific failure load (load per unit thickness) was enhanced from a value of about $60 \mathrm{~N} / \mathrm{mm}$ obtained for the glass to a maximum value of about $100 \mathrm{~N} / \mathrm{mm}$ for the GE composites and to a maximum of about $70 \mathrm{~N} / \mathrm{mm}$ for the GEF composite system. Similarly, the displacements at failure $(\delta)$ measured with a linear variable differential transformer (LVDT) were also found to be a strongly sensitive function of the type of reinforcement (GE or GEF) as well as the number of layers.
\end{abstract}

Keywords. Multilayer glass-epoxy material; biaxial flexure; reinforcement.

\section{Introduction}

The idea, that the brittle materials might be toughened by introducing a weak interface transverse to the propagating crack has been fully exploited in polymer composites. In such systems, the interface can be easily introduced by incorporating fibres, normally glass or carbon in a polymer matrix e.g. a resin. The success of this approach has led to a considerable effort being devoted currently to produce similar structures to toughen a completely new breed of ceramics, such as the laminated multilayer composites (Folsom et al 1994). The traditional layered structures promote either toughness by an interlayer crack deflection or strength by incorporating macroscopic residual stress for instance as in the laminar composites containing alternate layers of yttria stabilized tetragonal zirconia polycrystal (Y-TZP) and either alumina $\left(\mathrm{Al}_{2} \mathrm{O}_{3}\right)$ or a mixture of $\mathrm{Al}_{2} \mathrm{O}_{3}$ and yttria partially stabilized zirconia (Y-PSZ) (Wang and $\mathrm{Hu}$ 1996; Tomaszewski et al 1999). The other approach is lamination with a homogeneous layer to provide wear resistance and an alternating heterogeneous layer to provide toughness, viz. as has been demonstrated in the case of a silicon carbide based (SiC/SiC) system (Pender and Padture 1998). However, not much work has been done to understand the deformation process of glass-polymer multilayer composites (GPMLC) in biaxial flexure.

\footnotetext{
*Author for correspondence
}

Thus, the objectives of the present work were (a) fabrication of GPMLC and (b) to study their mechanical behaviour. The choice of glass as the matrix is guided by the fact that it is a model brittle material.

\section{Experimental}

Two different types of GPMLC samples were fabricated and compared with the failure behaviour of a thin, commercial soda-lime-silica glass cover slip of diameter $18 \mathrm{~mm}$ and thickness $0.3 \mathrm{~mm}$. These are laminar, multilayered (1, 2, 3 and 4 layered; designated respectively as $L 1, L 2, L 3$ and $L 4)$ glass-epoxy (GE) and glass-epoxy-Eglass fabric (GEF) composite structures. This was done to identify the best combination of the reinforcing elements in the GPMLC system. The epoxy was a commercial resin [Araldite Epoxy Resin, LY556, Ciba Speciality Chemicals (India) Ltd] with 15-20\% Hardner HY951 (Cibatul Ltd, India). The $E$-glass fabric was also commercially procured as a two-dimensional weave of $E$-glass fabric to an area density of $242 \mathrm{~g} \mathrm{~m}^{-2}$ (FGP Ltd, India). The multilayered structures were fabricated by alternate placement of glass and reinforcing layers by a hand lay-up technique followed by lamination at $\sim 25-50^{\circ} \mathrm{C}$ at a pressure of $\sim 2-$ $5 \mathrm{KPa}$. The density of the samples was measured gravimetrically while the volume fraction was measured by the standard, conventional method. The failure test of the glass sample as well as the GPMLC samples were then conducted using a universal testing machine (Instron, 
5500R) at a temperature of $30^{\circ} \mathrm{C}$, in a biaxial flexure mode (de With and Wagemans 1989) with a support ring of diameter $\sim 9 \mathrm{~mm}$ and a cross head speed (CHS) of $1 \mathrm{~mm} \mathrm{~min}{ }^{-1}$. The displacement at failure was measured with a linear variable differential transformer (LVDT) having a sensitivity of $0.7 \%$ of the full scale deflection.

\section{Results}

\subsection{Materials}

The volume fraction $\left(V_{\mathrm{fRP}}\right)$, total thickness of the reinforcing phase $\left(L_{\mathrm{t}}\right)$ and the thickness of the individual reinforcing layer $\left(L_{\mathrm{t}}\right)$ all increased with the number of layers $(n)$ of reinforcement in the GPMLC samples (figures 1-3). The variation of apparent density, $(D)$ with the number of layers is shown in figure 4 for the present samples. In the case of GE system, there was a slight enhancement in density with $n$. However, all samples in the GEF system had, in general, a much lower density than the matrix glass, irrespective of the number of layers. The volume fraction of reinforcing phase $\left(V_{\mathrm{fRP}}\right)$ and the total layer thickness $\left(L_{\mathrm{t}}\right)$ both increased with the number of reinforcing layers $(n)$ in the GPMLC samples (figures 1

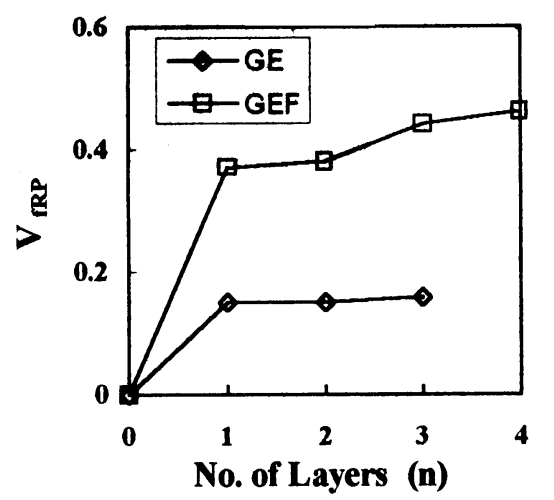

Figure 1. Variation of $V_{\mathrm{fRP}}$ with number of reinforcing layers in GPMLC samples.

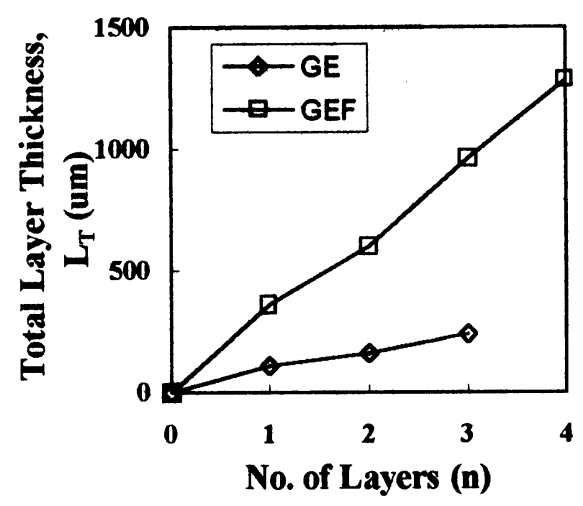

Figure 2. Variation of total layer thickness with number of reinforcing layers in GPMLC samples. and 2). However, the average thickness of individual layers $\left(L_{\mathrm{ti}}\right)$ in single, two layered $(L 2)$, three layered $(L 3)$ and four layered $(L 4)$ composite structures showed a slightly different trend (figure 3 ). $L_{\mathrm{ti}}$ initially increased with $n$ and then either decreased slightly (GE system) or remained somewhat insensitive to the number of layers (GEF system).

\subsection{Mechanical characterization}

The mechanical strength of the glass cover slip and the GPMLC samples was measured by means of the biaxial flexure test (de With and Wagemans 1989). The deformation process involved in failure of a glass cover slip was compared with that of GPMLC samples. The typical load $(P)$ vs load-point displacement $(d)$ behaviour of GPMLC samples (figures 5 and 6) shows a significant degree of non-linearity and hence, a distinct shift from the typical catastrophic, brittle failure behaviour. This definitely indicates the presence of a characteristic, pseudo-ductile feature in the load-deflection pattern of samples in both GE and GEF systems.

The specific failure load $\left(P_{\mathrm{f}} / T, T\right.$ the total thickness of samples) was higher for both the GPMLC samples in



Figure 3. Variation of individual layer thickness with number of reinforcing layers in GPMLC samples.

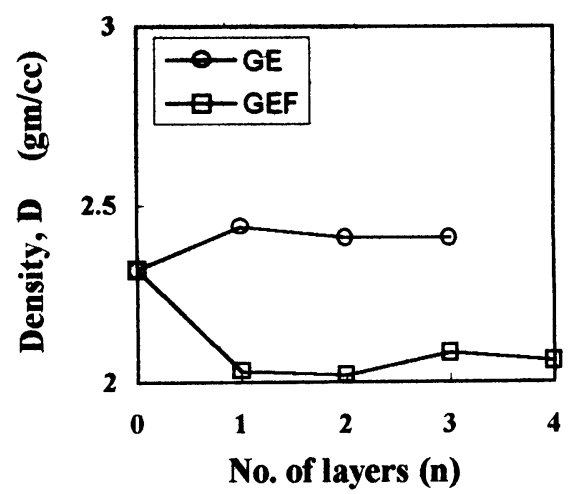

Figure 4. Variation of apparent density with number of reinforcing layers in GPMLC samples. 
comparison to that of the glass matrix (figure 7). From figure 7, it was evident that for a given system (GE and GEF), the specific failure load also increased with the number of layers, however, the property improvement was more marked for the GE samples than the GEF samples. The load point displacement for the samples also exhibited a similar behaviour (figure 8).

The catastrophic failure pattern of the matrix glass disk in biaxial flexure tests (figure 9) was typical of brittle fracture. In contrast, the GPMLC samples showed a

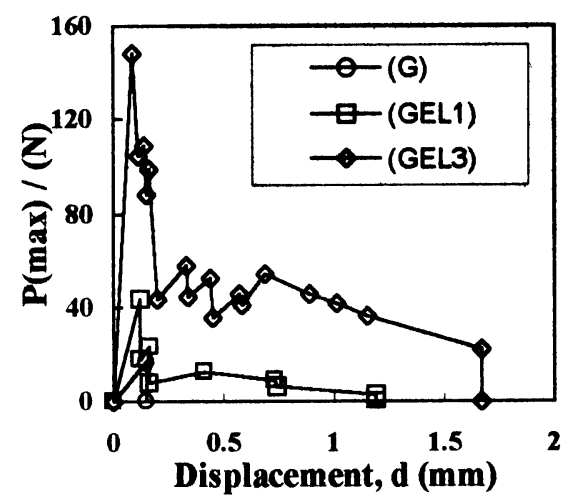

Figure 5. Typical load-displacement behaviour of GE composite samples.

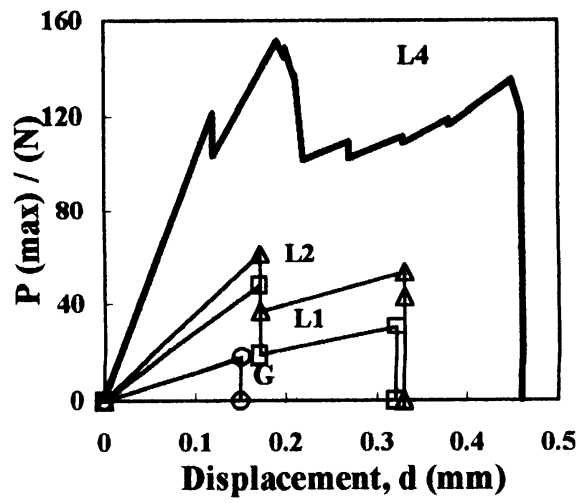

Figure 6. Typical load-displacement behaviour of GEF composite samples.

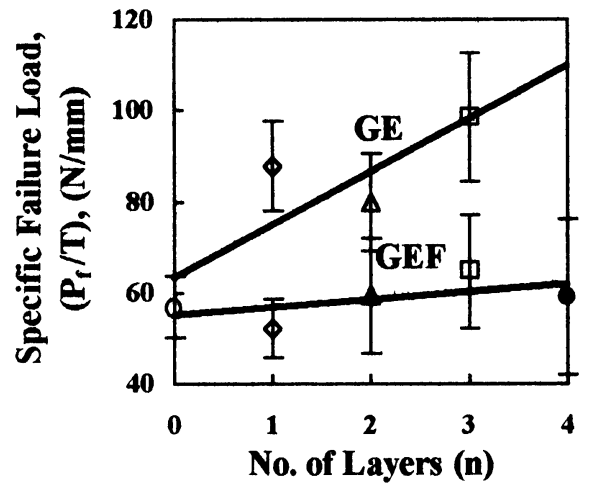

Figure 7. Variation of specific failure load with number of reinforcing layers in GPMLC samples. pseudo-ductile behaviour (figures 5 and 6) as reflected through property enhancement (figure 7). However, the improvement in the case of the GEF system was not as much as would be otherwise expected. This could be linked to the presence of interfacial cracks (figure 10) in the GEF samples. The improvement in specific failure load of the GPMLC samples (both GE and GEF systems) is likely to be linked to the constrained growth of ring

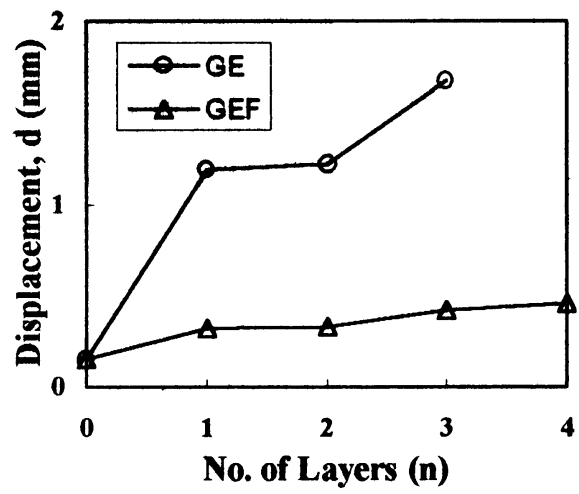

Figure 8. Variation of load point displacement with number of reinforcing layers in GPMLC samples.

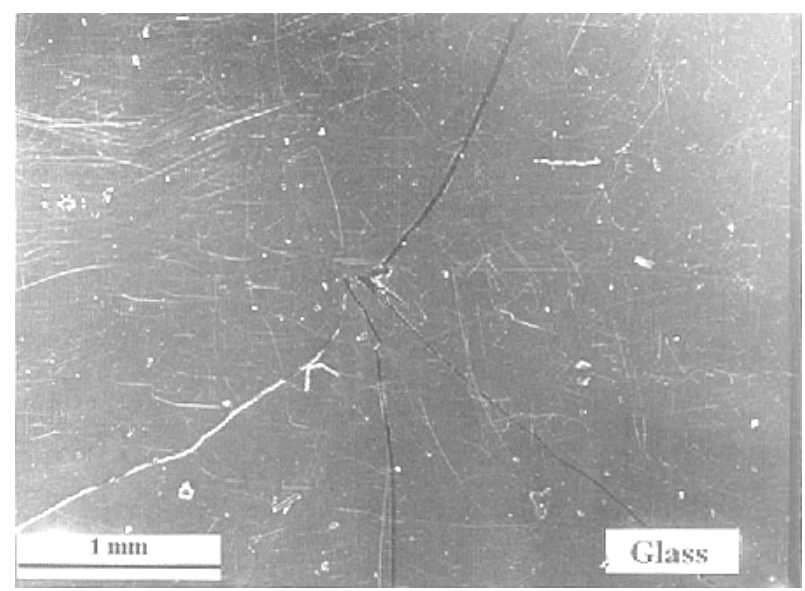

Figure 9. Fracture surface of glass cover slip.



Figure 10. Interfacial crack in the GEF sample. 

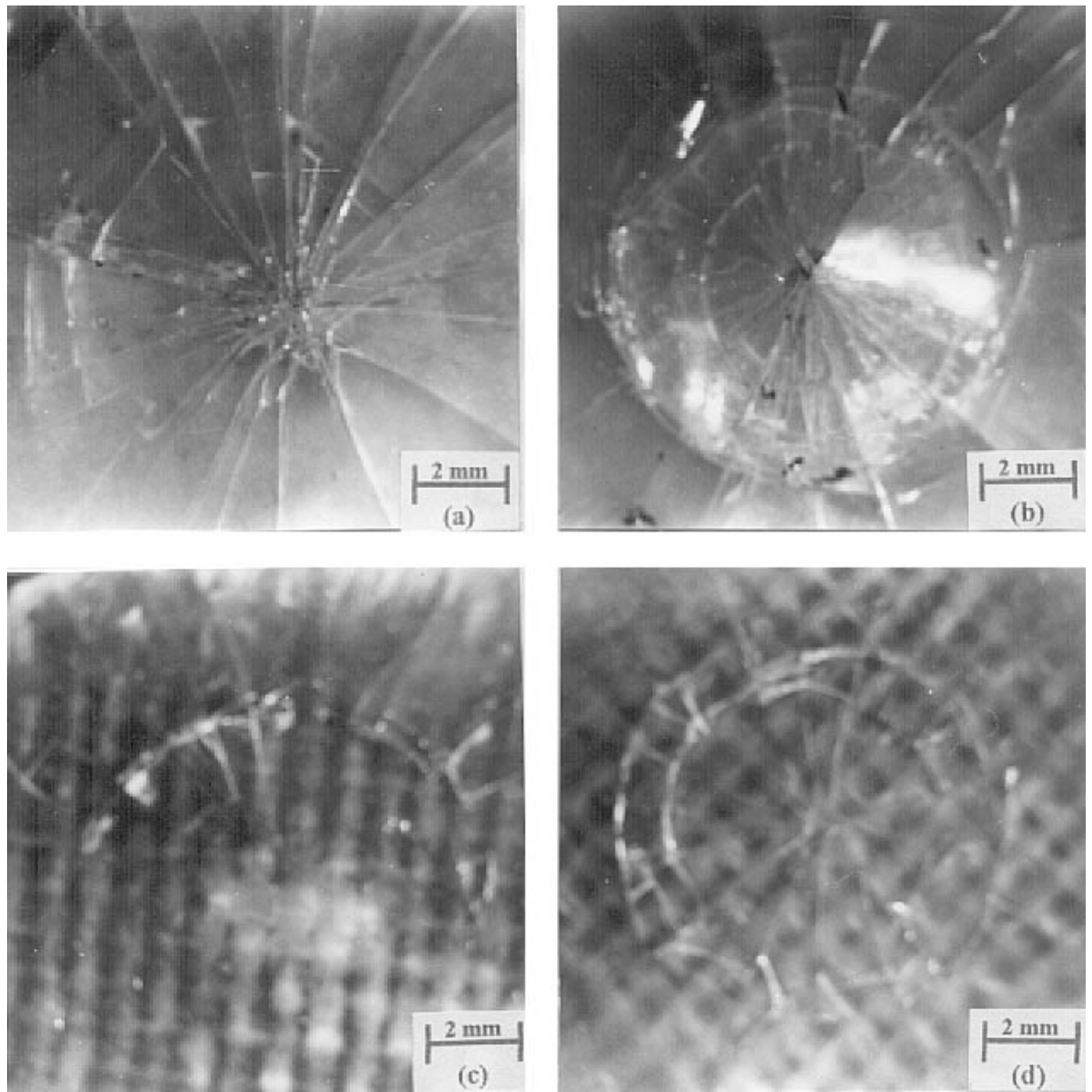

Figure 11. Tensile side of the fracture surface of samples in the various GPMLC systems: (a) GE, single layer (GEL1), (b) GE, triple layer (GEL3), (c) GEF, single layer (GEFL1) and (d) GEF, triple layer (GEFL3).

cracks through the reinforcing phase (figures $11 \mathrm{a}-\mathrm{d}$ ). For a given system [GE (figures 11a, b) or the GEF (figures $11 \mathrm{c}, \mathrm{d})]$, the more the number of layers, more was the number of ring cracks and hence, possibly the energy dissipative process became more active. The growth of the ring cracks can be constrained due to the presence of the reinforcing epoxy resin which may cause crack blunting (figure 11b). In addition, the presence of the woven fabric in the GEF system could also cause crack deflection (figure 11d). All such energy dissipative processes would enhance the specific failure load (figure 7).

\section{Conclusions}

The comparative behaviour of the deformation processes involved in the failure of commercial soda-lime-silica glass disks $(G)$ and multilayered glass disk-epoxy (GE) as well as glass disk-epoxy-E-glass fabric (GEF) composite structures has been studied in the present work. It was found that depending on the number of layers, the specific failure load could be enhanced from a value of about $60 \mathrm{~N} / \mathrm{mm}$ obtained for the glass to a maximum value of about $100 \mathrm{~N} / \mathrm{mm}$ for the GE composites and to a maximum of about $70 \mathrm{~N} / \mathrm{mm}$ for the GEF composite system. In addition, the presence of a characteristic pseudo-ductile behaviour was exhibited by samples from both GE and GEF systems. The results obtained from optical microscopy seem to indicate that in the GPMLC structures, in general, the crack evolution resembled that of the constrained growth of a ring crack through the laminated structure consisting of alternating layers of a brittle material (glass) and a reinforcing phase (epoxy in the GE 
system or epoxy plus $E$-glass fabric in the GEF system). This was in contrast to the typical brittle failure pattern observed for the thin glass disks broken in biaxial flexure. However, the scanning electron microscopic evidence possibly indicated that the lack of improvement in the mechanical property e.g. specific failure load of the GEF system could be linked to the presence of interfacial cracks at the glass-epoxy interface, which, in principle, should provide a sight for a quicker debonding to occur.

\section{Acknowledgements}

The financial support received in the form of a Senior Research Fellowship and in the form of a Project funding by the Department of Science and Technology (DST), New Delhi are gratefully acknowledged by AS and AKM, respectively. Dr S Mandal, Refractories Division, and Mrs A Laskar, Scanning Electron Microscopy Division, Central Glass and Ceramic Research Institute, Kolkata are thanked for the experimental assistance provided in optical microscopy and scanning electron microscopy, respectively.

\section{References}

Folsom C A, Zok F W and Lange F F 1994 J. Am. Ceram. Soc. 77 689, 2081

Pender D C and Padture N 1998 J. Mater. Sci. Lett. 17 999

Tomaszewski H, Strzeszewski J and Gebicki W 1999 J. Eur. Ceram. Soc. 19255

Wang H and Hu X 1996 J. Am. Ceram. Soc. 79553

de With G and Wagemans H H M 1989 J. Am. Ceram. Soc. 72 1538 